\title{
Camera-based Bidirectional Reflectance Measurement for Road Surface Reflectivity Classification
}

\author{
Martin Roser and Philip Lenz \\ Department of Measurement and Control \\ Karlsruhe Institute of Technology \\ D-76131 Karlsruhe, Germany \\ Email: roser@kit.edu,philip.lenz@kit.edu
}

\begin{abstract}
In this paper we propose a novel framework for road reflectivity classification in cluttered traffic scenarios by measuring the bidirectional reflectance distribution function of road surfaces from inside a moving vehicle. The predominant restrictions in our application are a strongly limited field of observations and a weakly defined illumination environment. To overcome these problems, we estimate the parameters of an extended Oren-Nayar model that considers the diffuse and specular behavior of real-world surfaces and extrapolate the surface reflectivity measurements to unobservable angle combinations. Model ambiguities are decreased by utilizing standardized as well as customized reflection characteristics. In contrast to existing approaches that require special measurement setups, our approach can be implemented in vision-based driver assistance systems using radiometrically uncalibrated gray value cameras and GPS information. The effectiveness of our approach is demonstrated by a successful classification of the road surface reflectance of expressway scenes with low error rates.
\end{abstract}

\section{INTRODUCTION}

Digital imaging devices provide a dense environment perception and their benefits are already exploited exhaustively in vision-based driver assistance systems (DAS). Whereas many state-of-the-art computer vision algorithms work reliably in good weather, they are heavily affected by adverse conditions such as reflective road surfaces in combination with disadvantageous illumination scenarios (see Fig. 1). However, proper and robust operation in arbitrary situations is a security-relevant prerequisite to many applications, particularly on board mobile vehicles. Hence, estimation of road surface reflection properties is fundamental for improving vision-based DAS. It may allow for evaluating the quality of environment perception to the point of completely compensating the reflective part of the luminance distribution, only leaving the road surface albedo.

The bidirectional reflectance distribution function (BRDF) is the predominant radiometric concept used in computer vision. The difficulty in deriving a BRDF is to acquire a sufficient number of measurements in a well-defined imaging and illumination setup. So far, this can only be performed experimentally using sophisticated measurement devices. Identifying the road surface reflectance from inside a moving vehicle using only a single grayscale image is highly illposed and challenging for several reasons:

1) Recent vision-based DAS use low-dynamic-range cameras with radiometrically uncalibrated CCD sensors.
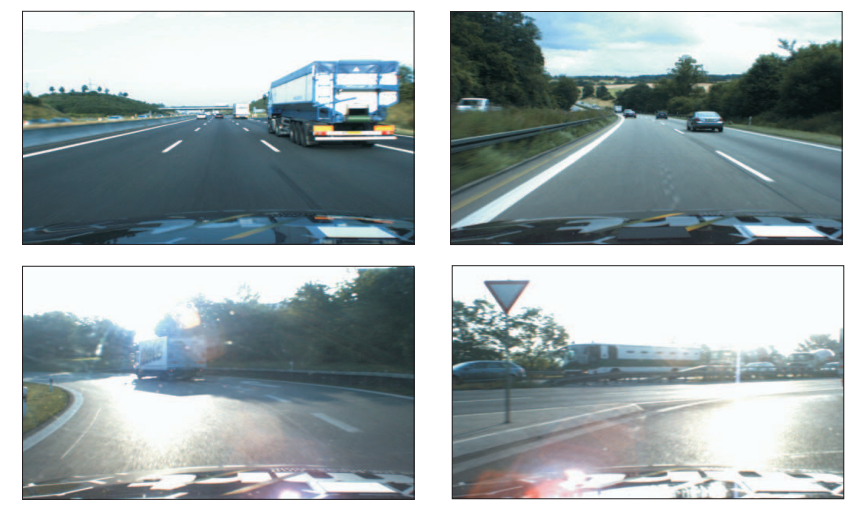

Fig. 1. Road Surface Reflectivity. Diffuse road surfaces (top row) provide good visibility of road markings and traffic participants, whereas specular surfaces (bottom row) are a challenging problem for current DAS.

2) Sensor data from complex traffic scenes in adverse weather conditions is subject to severe noise which lowers measurement quality.

3) In-vehicle camera perspectives only yield sparse measurements from flat observation angles (see Fig. 6). Hence, only a small part of the road surface BRDF is observable, which makes accurate identification of the general reflection characteristic hard.

4) Unknown illumination directions do not allow for a systematic control of measurement acquisition, like it is the case e.g. in gonioreflectometers.

This paper addresses the problem of estimating the road surface BRDF in cluttered real-world traffic scenarios. Our main contribution is a framework for BRDF estimation and road surface reflectivity classification using only a single grayscale image and GPS information acquired from inside a moving vehicle. A core part of our approach is to fit measured BRDF values to an appropriate physical BRDF model in order to extrapolate the surface reflectivity measurements from a considerably limited field of observation. Based on the estimated BRDF model, we propose meaningful reflectance features that allow for an accurate identification of the road reflectivity state. The effectiveness of our approach is demonstrated quantitatively by a binary classification of expressway scenes into the classes diffuse and specular (example images for these classes are depicted in Fig. 1 and Fig. 7). 
Section II reviews relevant literature. The BRDF definition is summarized in Section III. Section IV introduces an extended Oren-Nayar reflectance model, that we are using for achieving a general BRDF characteristic of the surface patch only from sparse measurements. Section $\mathrm{V}$ proposes the novel framework for BRDF estimation and classification of the road surface reflectivity. We present detailed feature evaluation and classification results in Section VI and conclude our work in Section VII.

\section{RELATED WORK}

The BRDF offers a pointwise description of light reflection on real surfaces. It can be measured by acquiring dense samples taken from well-defined observer and light source positions. Traditional gonioreflectometer-based approaches [27], [4], [15] demand flat material samples and take up to several hours for data acquisition. Image-based measurement systems [13], [14], [10] are proposed in order to speed up acquisition time at comparable accuracy. However, they all demand a well-defined experimental setup and are still quite time and memory-consuming.

Extensive databases [4], [14] provide dense BRDF measurements of real-world materials. However, the materials are typically chosen to span a wide range of photometric properties for computer graphics rendering. BRDF measurements of road samples are rare. Although Meister [15] evaluated several urban surfaces for identifying land covers in satellite supported sensor data, only a small amount of his data such as asphalt and concrete is relevant for automobile applications. Hence, an assignment of acquired road surface BRDF measurements to available database knowledge is unfeasible.

In order to improve BRDF applicability, the general goal is to find an accurate low-parameter representation of an already densely sampled BRDF. To this end, the measurements are typically fitted to an appropriate analytical model. They can basically be divided into empirical BRDF models [2], [21], [27] and physical models that simulate the microscopic surface geometry [22], [3], [18], [19], [16]. Empirical functions describe densely measured BRDF data well, whereas physical models are also capable of fitting noisy and sparse BRDF measurements of real surfaces, which is the task in our application.

In the context of automobile applications, BRDF measurements are predominantly used for optimizing the road illumination level and traffic visibility [11], [8], using standardized reflection parameters [1] for robust surface characterization. Other efforts have attempted to improve headlamp design, preventing dazzle effects from the driver or oncoming traffic [26], [25]. However, they only evaluate a few surfaces in laboratory environments or with specialized field measurement equipment. A generic approach for road surface reflectivity classification has to cover a multitude of road surfaces, subject to arbitrary wetting conditions. Hence, any database generation in laboratory environments will fail due to the enormous amount of data that has to be acquired.

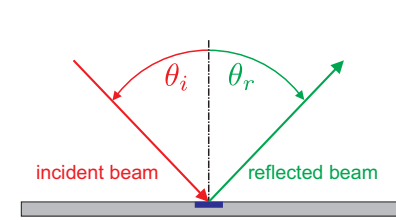

(a) Perfect mirror.

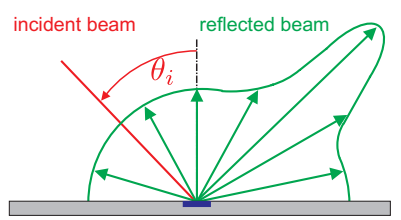

(b) Arbitrary surface.
Fig. 2. Reflected radiance. On a mirror-like surface, the incident light ray is totally reflected (a). Dealing with arbitrary surfaces, any combination of diffuse or specular reflection may occur (b). Note, that only light rays in the plane of incidence are visualized.

\section{BRDF DEFINITION}

The reflectance of opaque objects usually depends on the angles at which its surface is illuminated and observed. For a perfect mirror the zenith angle $\theta_{i}$ of an incident light ray equals the zenith angle $\theta_{r}$ of a reflected light ray as depicted in Fig. 2(a). For an arbitrary surface patch, any combination of diffuse or specular reflection may occur and lead to light reflections emitting in all directions of the upper hemisphere (Fig. 2(b)).

In general, this behavior can be described in terms of the BRDF. According to [17], the BRDF $f_{r}$ of a surface $d A$ can be defined as the ratio of reflected radiance $L_{r}$ and incident irradiance $E_{i}$ for all possible observer and light source positions given by their azimuth $\left(\phi_{r}, \phi_{i}\right)$ and zenith angles $\left(\theta_{r}, \theta_{i}\right)$ :

$$
f_{r}\left(\phi_{i}, \theta_{i}, \phi_{r}, \theta_{r}\right)=\frac{d L_{r}\left(\phi_{r}, \theta_{r}\right)}{d E_{i}\left(\phi_{i}, \theta_{i}\right)}
$$

The incident irradiance $E_{i}$ describes the radiant flux $\Phi$ per unit area $A$

$$
E_{i}=\frac{d \Phi}{d A}
$$

and the reflected radiance $L_{r}$ is given

$$
L_{r}=\frac{d^{2} \Phi}{d A \cos \theta_{r} \mathrm{~d} \Omega},
$$

which is the radiant flux $\Phi$ per unit solid angle $\Omega$ and a surface perpendicular to the propagation direction.

\section{REFlECTANCE MODEL}

Reflectance models are typically introduced in order to achieve low-parameter representation of the acquired BRDF measurements. One prominent physical BRDF model for diffuse reflection is proposed by Oren and Nayar [18], [19], [16]. It assumes isotropic surfaces that are composed of small surface facets arranged in v-shaped grooves of constant width $d$ as depicted in Fig. 3. Superimposition of the Lambertian behavior of all surface facets leads to two diffuse reflection components. $f_{r}^{d, d i r}$ includes all directly reflected parts and $f_{r}^{d, m s}$ takes multiple reflections into account:

$$
\begin{aligned}
f_{r}^{d, d i r}=\frac{\rho}{\pi}\left(C_{1}(\right. & \left.k_{w}\right)+C_{2}\left(k_{w}\right) \cos \phi \tan \theta_{2} \\
& \left.+C_{3}\left(k_{w}\right)\left(1-|\cos \phi| \tan \frac{\theta_{1}+\theta_{2}}{2}\right)\right),
\end{aligned}
$$




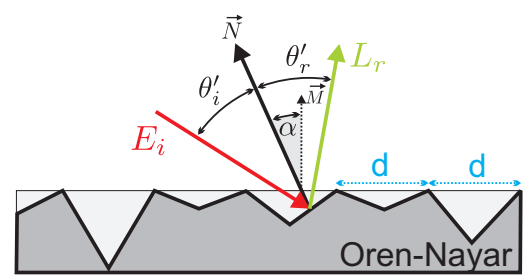

Fig. 3. Surface modeling. Oren-Nayar assumes a macroscopic surface consisting of small surface facets, arranged in $\mathrm{v}$-shaped grooves of constant width $d$.

$$
f_{r}^{d, m s}=0,17 \frac{\rho^{2}}{\pi} \frac{k_{w}^{2}}{k_{w}^{2}+0,13}\left(1-\frac{4 \theta_{2}^{2}}{\pi^{2}} \cos \phi\right),
$$

where $\rho$ is the surface albedo, $\phi=\left|\phi_{i}-\phi_{r}\right|$ is the relative azimuth angle, $\theta_{1}=\max \left(\theta_{i}, \theta_{r}\right)$ is the maximum zenith angle and $\theta_{2}=\min \left(\theta_{i}, \theta_{r}\right)$ is the minimum zenith angle, respectively. $C_{1}\left(k_{w}\right), C_{2}\left(k_{w}\right)$ and $C_{3}\left(k_{w}\right)$ are constants that are solely dependent on $k_{w}$ and the geometric relation between the observer and light source [19]. For clarity, all model equations do not explicitly state any dependency from the observer and light source positions $\theta_{i}, \theta_{r}, \phi$.

Real-world road surfaces demand for an accurate description of surface reflections which cannot be regarded in the traditional Oren-Nayar model. Therefore, the traditional Oren-Nayar model is extended by a specular part [15], [22], where all surface facets are assumed to act as small, perfect mirrors. Specular reflections only occur in the direction of total reflection for each facet. This yields the local illumination angle $\theta^{\prime}=\theta_{r}^{\prime}=\theta_{i}^{\prime}$ with respect to the surface facet normal $\vec{N}$ (see Fig. 3), that can be determined geometrically [24]. We model the probability distribution $P$ of the surface facet normals that are involved in the specular reflection using a zero-mean Gaussian distribution function with standard deviation $k_{w}$ :

$$
P\left(k_{w}\right) \propto \mathrm{e}^{\frac{\alpha^{2}}{2 k_{w}^{2}}}
$$

where $\alpha$ is the zenith angle of the surface facet normal $\vec{N}$ with respect to the macroscopic surface normal $\vec{M}$ as illustrated in Fig. 3. Using (6), the specular part can be described as

$$
f_{r}^{s}\left(k_{w}, n\right)=\frac{F(n) P\left(k_{w}\right) G}{4 \cos \theta_{i} \cos \theta_{r}}
$$

where $F(n)$ denotes the Fresnel reflectance that describes the photometric behavior of light rays on surfaces [7] and $G$ is a geometric attenuation factor regarding masking and shadowing effects within the facet structure [19].

Finally, a weighted superposition of the diffuse and specular reflection parts $\left(f_{r}^{d}\right.$ and $\left.f_{r}^{s}\right)$ gives an extended Oren-Nayar $\left(O N_{\text {ext }}\right)$ model:

$$
f_{r}=k_{d} f_{r}^{d}\left(k_{w}\right)+k_{s} f_{r}^{s}\left(k_{w}, n\right),
$$

where $k_{d}$ and $k_{s}$ denote the weighting factors for the diffuse and specular reflection terms, $n$ the material refraction index and $k_{w}$ is a parameter for the surface roughness.

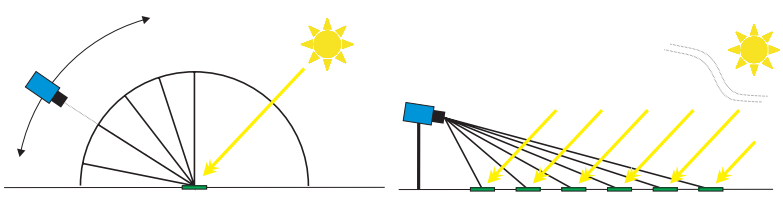

(a) Traditional BRDF measure- (b) Vehicle based BRDF measurement devices. ment approach.

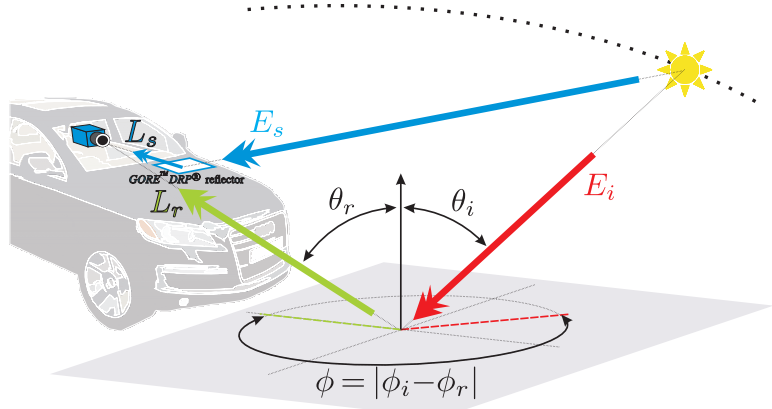

(c) Automotive BRDF measurement setup

Fig. 5. Experimental setup. Whereas traditional BRDF measurement devices, such as gonioreflectometers, observe the same surface patch under varying observer and light source positions (a), we observe different surface patches for each perspective that are assumed to have an invariant reflectance behavior (b). (c) shows a three-dimensional geometric model of our automotive BRDF measurement setup.

\section{REFLECTANCE ESTIMATION FRAMEWORK}

A robust road surface reflectivity classification technique depends on reliable, strong BRDF features. Single images taken from an in-vehicle camera, in principle, provide sufficient information for that task.

In this section, we propose a novel framework for visionbased road surface reflectivity classification as depicted in Fig. 4. First, we determine all radiometric and geometric quantities that are necessary for BRDF measurement acquisition according to (1) (Fig. 4(a-b)). The BRDF measurements are acquired within $10 \times 10 \mathrm{~cm}$ patches on the road surface (Fig. 4(c)). Then, we fit an extended Oren-Nayar BRDF model to the acquired data and extract reliable BRDF features (Fig. 4(e-f)). Finally, these features form a lowdimensional description vector that is used to decide on the road surface reflectivity class (Fig. 4(d)).

\section{A. Data Acquisition}

In order to compute the BRDF measurements according to (1), the incident irradiance $E_{i}$ on the road surface, the reflected radiance $L_{r}$ towards the viewer as well as the geometric observer and light source positions specified by $\theta_{i}, \theta_{r}, \phi$ must be known. These entities can be acquired from our measurement setup as depicted in Fig. 5(c).

A predefined camera mounting behind the windshield as well as knowledge about the camera orientation, received from two GPS antennas, specify one side of the geometric relationship between observer and light source. Assuming the sun as the only relevant illuminant, the latter half can be computed using a formula for the momentary, geocentric sun position [23]. 


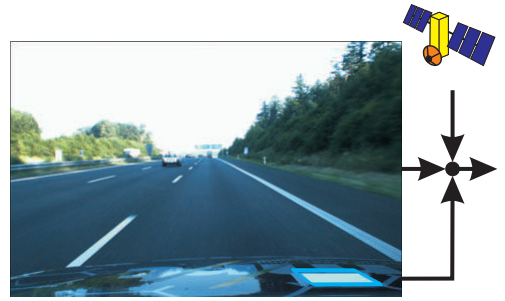

(a) Input information.

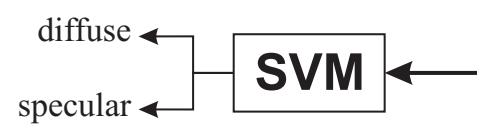

(d) Road surface reflectivity classification.

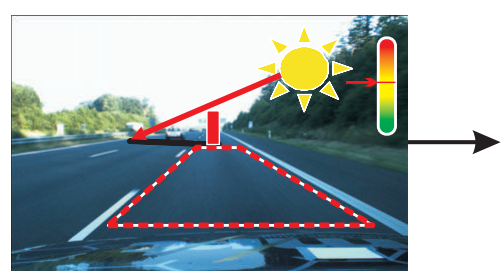

(b) Defined experimental setup.

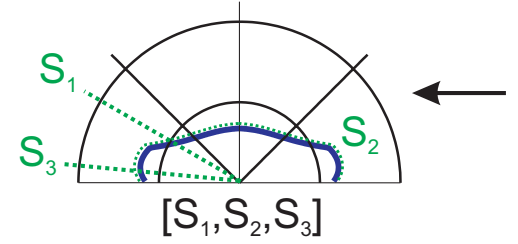

(e) Robust feature extraction.

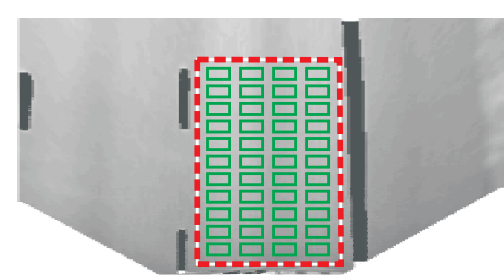

(c) Road reflectance measurement.

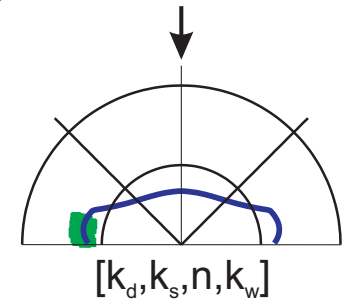

(f) BRDF model fitting.

Fig. 4. Flowchart of proposed method. (a) An in-vehicle camera, two GPS antennas and a diffuse reflector provide all necessary input information for a well-defined, experimental measurement setup. In (b), the illumination direction is illustrated by introducing an artificial $2 \times 0.5 \mathrm{~m}$ object and computing its shadow with respect to the current sun position. The relative sun intensity is shown by a color bar in the right top corner of (b). In (c), the BRDF measurements are acquired within a $10 \times 10 \mathrm{~cm}$ neighborhood (green boxes). In (f), an extended Oren-Nayar BRDF model is fitted to the received measurements. Then, robust features (e) are used for a SVM classification of different road reflectivity states (d).

During the image acquisition process, each camera pixel integrates the radiant flux $\Phi$ that is striking its area $A$ over a defined exposure time $T_{e}$. Given a linear camera sensor characteristic, disregarding any noise stemming from the image acquisition process and using (2), the relation between a camera pixel's gray value $g$ and its incident irradiance $E$ yields

$$
g \propto \int_{0}^{T_{e}} A E d t
$$

According to [9], the relation between the reflected radiance $L_{r}$ of a road surface patch and its incident irradiance $E$ on the camera sensor is given by

$$
L_{r} \propto \frac{E}{\cos ^{4} \theta},
$$

with $\theta$ defining the relative, geometric position between camera and reflector. Note, that the proportionality constants of both equations solely depend on camera properties. Hence, a pointwise description between the radiance $L_{r}$ reflected from the road surface patch and the gray value $g$ of the camera pixel can be deduced from (9) and (10). In contrast to traditional BRDF measurement techniques that observe the same material patch from different observer positions (see Fig. 5(a)), we assume the road surface to have an invariant BRDF characteristic and observe its radiance from different angles in a single image as depicted in Fig. 5(b). The radiance measurements are achieved from a birds-eye view perspective by evaluating the mean gray value in a $10 \times 10 \mathrm{~cm}$ neighborhood of $75 \times 30$ supporting points as illustrated in Fig. 4(c).

For determining $E_{i}$ on the road surface, we assume that the sun irradiance is constant throughout the vehicle surrounding. This enables an estimation of the incident irradiance on the road surface $E_{i}$ by determining the incident irradiance $E_{s}$ on a diffuse $G O R E^{T M} D R P^{\circledR}$ reflector that is mounted on the hood of our experimental vehicle as depicted in Fig. 5(c). The BRDF equation of a perfectly diffuse reflector is given by

$$
L_{s}=\frac{\rho}{\pi} E_{s}
$$

For robustness, $E_{s}$ is computed according to (9)-(11) by taking the mean of all gray values $\bar{g}$ exceeding a certain threshold in the gray value histogram of the reflector. Hence, the incident irradiance on the road surface $E_{i}$ yields

$$
E_{i}=E_{s}=K \cdot \frac{\bar{g}}{T_{e}},
$$

where the proportionality constant $K$ can be determined, using the camera and reflector properties as well as their relative, geometric position. Note, that $K$ remains constant for all images captured with the same experimental setup.

\section{B. Preprocessing}

The reflected surface radiance is determined from intensity values of a camera sensor. Hence, major changes in the road surface albedo such as bright road markings on dark concrete violate the assumption of invariant road surface patches and lead to outliers in the BRDF measurements. Moreover, all atmospheric effects between the surface patch and the camera sensor, for example raindrops on the car windshield, will additionally disturb the measurement process. Since this approach should enhance DAS especially in adverse weather conditions, insensitivity towards these effects is of particular importance.

For this reason, we use a previously developed visionbased raindrop detection [6], [20] as well as a standard lane detection approach and consider only BRDF measurements that are not disturbed by raindrops and do not lie on road markings. 


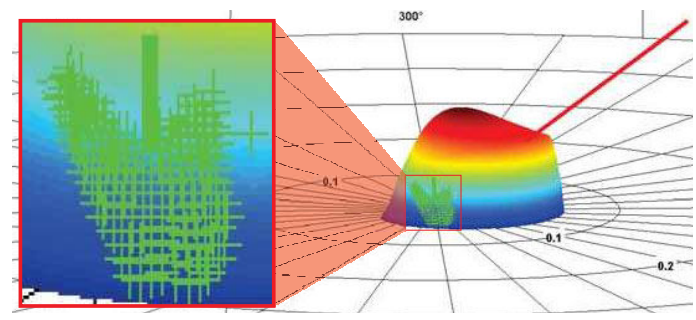

Fig. 6. Model fitting. An extended Oren-Nayar model function (colored envelope) is fitted to few BRDF measurements (green crosses), received from a strongly limited field of observation.

\section{BRDF Model Fitting}

A meaningful BRDF evaluation of the road surface can only be realized for a bounded area in front of the vehicle as illustrated in Fig. 4(b). This corresponds to a zenith angle range of $77^{\circ}<\theta_{r}<87^{\circ}$ and an azimuth angle $\phi_{r}$ that is limited to the car heading $\pm 20^{\circ}$. Since this results in a limited field of view (see Fig. 6), the light source and viewer positions are certainly different for various daytimes and vehicle headings and a comparison of different traffic scenes cannot be performed on the BRDF measurements, directly. Therefore, we fit an extended Oren-Nayar model in order to achieve a model-based extrapolation of the whole BRDF of the road surface. Applying standard nonlinear LevenbergMarquardt optimization [12], we estimate the unknown $\mathrm{ON}_{\text {ext }}$ parameters $\left(k_{w}, k_{s}, k_{d}, n\right)$, conditioning normalized weighting factors $\left(k_{d}+k_{s}=1\right)$ [3].

Note, that traditional BRDF model fitting tries to find a low-parameter approximation of an already densely sampled BRDF in order to perform a model-based interpolation between the acquired samples. The estimated model parameters themselves normally do not play a major role. In contrast to that, our purpose is estimating a BRDF parametrization from a strongly limited field of observation as depicted in Fig. 6.

\section{Feature Extraction}

In addition to the estimated $\mathrm{ON}_{\text {ext }}$ model parameters, we extract robust CIE reflectance parameters $S_{1}, S_{2}$ according to [11], [1], but for an adjusted observer position $\theta_{r} \approx 82^{\circ}$. These parameters evaluate the whole BRDF function for two characteristic points:

$$
\begin{gathered}
S_{1}=\frac{f_{r}\left(\phi=180^{\circ}, \theta_{i}=26.6^{\circ}, \theta_{r} \approx 82^{\circ}\right)}{f_{r}\left(\phi=180^{\circ}, \theta_{i}=0^{\circ}, \theta_{r} \approx 82^{\circ}\right)}, \\
S_{2}=\frac{Q_{0}}{f_{r}\left(\phi=180^{\circ}, \theta_{i}=0^{\circ}, \theta_{r} \approx 82^{\circ}\right)},
\end{gathered}
$$

with

$$
Q_{0}=\frac{1}{\Omega} \int_{(\Omega)} f_{r}\left(\theta_{i}, \theta_{r}, \phi\right) \mathrm{d} \omega .
$$

$S_{2}$ determines the enclosed BRDF volume $Q_{0}$ with respect to the reflectance in surface normal direction. Especially $S_{1}$ is a customized reflectance parameter for headlight and street lightning design, that indicates the reflectance for a standard light source position $\phi=180^{\circ}$ and $\theta_{i}=26.6^{\circ}$.
We expect, that this angle combination is not ideal for our application. Therefore, we propose a novel reflectance feature $S_{3}$ :

$$
S_{3}=\frac{f_{r}\left(\phi, \theta_{i}, \theta_{r} \approx 82^{\circ}\right)}{f_{r}\left(\phi=180^{\circ}, \theta_{i}=0^{\circ}, \theta_{r} \approx 82^{\circ}\right)},
$$

which is computed using the current vehicle heading and sun position that can be obtained as discussed in Section V-A.

\section{E. Classification}

We choose selected combinations of $\mathrm{ON}_{\text {ext }}$ model parameters $k_{w}, k_{d}, n$ and reflectance features $S_{1}, S_{2}, S_{3}$ to create a descriptor vector $\mathbf{v}$, which is then used to decide on the road surface reflectivity class. In our case, we perform a binary classification into the classes $C=\{$ diffuse, specular $\}$. Thus, the problem of classification can be thought of as finding some function $f$ that maps $\mathbf{v}$ from descriptor space $\mathcal{D}$ into the classes $C$ with $c=f(\mathbf{v})$, where $f: \mathcal{D} \rightarrow C$.

Numerous machine learning methods have been proposed for finding such a function $f$ from training examples [5] using techniques like k-Nearest-Neighbor, Decision Trees, Neural Networks and Support Vector Machines (SVM). As SVMs are simple, fast, and powerful, we decided to use them as our learning and classification method.

One of the advantages of SVM's is that kernel methods, such as linear and RBF (Radial Basis Functions), can be incorporated in the algorithm. With them, non-linear decision boundaries can be found. However, since one parameter for the RBF kernel has to be optimized manually and linear separation already gives satisfactory results, we preferred applying a linear kernel.

\section{RESULTS}

For all experiments, we used 680 grayscale images with a resolution of $1024 \times 768$ pixels. They observe different illumination situations and road surfaces on expressways, mainly covering specular and diffuse reflections on dry and damp roads (see Fig. 1 and Fig. 7).

The BRDF models are visualized in a spherical coordinate system, using the so called $q$-body representation [11]: For a fixed light source, a set of vectors, given by their zenith and azimuth angles to the observer and the corresponding BRDF value as their vector norm, composes an envelope that is describing the surface reflection characteristic (for examples, refer to Fig. 7). For better illustration, some figures also show 2D cuts of these 3D BRDF envelopes.

In order to overcome the problem of limited image data for the classification task, we performed a 5-fold-crossvalidation. We ensured that no image is used for both training and testing at the same time, as well as we ensured equal amount of images for each set.

\section{A. BRDF Model Estimation}

As depicted in Fig. 7, the extended Oren-Nayar models and their parameters allow for successful distinction between different road reflectivity conditions for well-posed test image examples. However, real-world traffic scenes are subject to measurement noise, especially in adverse weather 
conditions. Furthermore, estimating a BRDF model that describes the material reflectance for all possible observer and light source positions from a strongly limited field of observation as illustrated in Fig. 6 is a highly ill-posed problem. Our experiments show, that for these reasons, the $\mathrm{ON}_{\text {ext }}$ model parameters alone are not sufficient to classify the road surface reflection state accurately.

\section{B. Model Ambiguities \& Measurement Noise}

We introduced a data preprocessing for reducing measurement noise and extracted more robust reflectance features $S_{1}, S_{2}, S_{3}$ for increasing model uniqueness.

In order to illustrate this phenomenon of model ambiguities, we parametrized an arbitrary BRDF $q$-body and varied the $\mathrm{ON}_{\text {ext }}$ parameters $k_{d}$ and $k_{w}$. The refraction index $n$ is assumed to be constant for this experiment and the specular weighting factor is set to $k_{s}=1-k_{d}$. The shape similarity between the original q-body and the varied ones is measured in terms of the Sum-of-Squared Differences (SSD). As depicted in Fig. 8(a), various parameter sets lead to small SSD errors (red line), that means they all describe similar $q$ bodies. Fig. 8(d) shows 2D cuts of two exemplary q-bodies for diffuse and specular road surfaces. Although the diffuse BRDF models (and specular BRDF models, respectively) show similar shapes, their $\mathrm{ON}_{\mathrm{ext}}$ parameters differ considerably. However, the proposed reflectance features $S_{1}, S_{2}, S_{3}$ are more stable and hence are invariant to model ambiguities.

Fig. 8(b) shows the original analysis area with and without road markings. As illustrated in Fig. 8(e), road markings significantly influence the BRDF estimation. However, only considering BRDF measurements that do not lie on road markings lead to an improved BRDF estimation. Note, that the ground truth in Fig. 8(e) is achieved by manually shrinking the analysis area in such way that it is not affected by road markings anymore.

Due to the BRDF model restrictions, an inherent robustness to raindrops already exists. We tested this statement by estimating the BRDF directly before and after wiper passing. As depicted in Fig. 8(f), the estimated BRDF functions are very similar, even if the image is heavily distorted by raindrops on the windshield (see Fig. 8(c)). Considering only image regions that are not affected by raindrops [6], [20] further improves the results.

\section{Surface Reflectivity Classification}

The road surface reflectivity was classified using selected combinations of either the extended Oren-Nayar model parameters $\left(n, k_{d}, k_{w}\right)$ or the robust reflectance features $\left(S_{1}, S_{2}, S_{3}\right)$. The resulting error rate of the different combinations is shown in Fig. 9. Table I depicts the confusion matrices for selected feature combinations.

A decision about the reflectance class with $\mathrm{ON}_{\mathrm{ext}}$ parameters results in a high error rate between $18.5 \%\left(k_{d}\right)$ and $24.7 \%\left(k_{d}, k_{w}, n\right)$. In constrast, the proposed reflectance features outperform a classification based on the extended Oren-Nayar model parameters, exclusively.

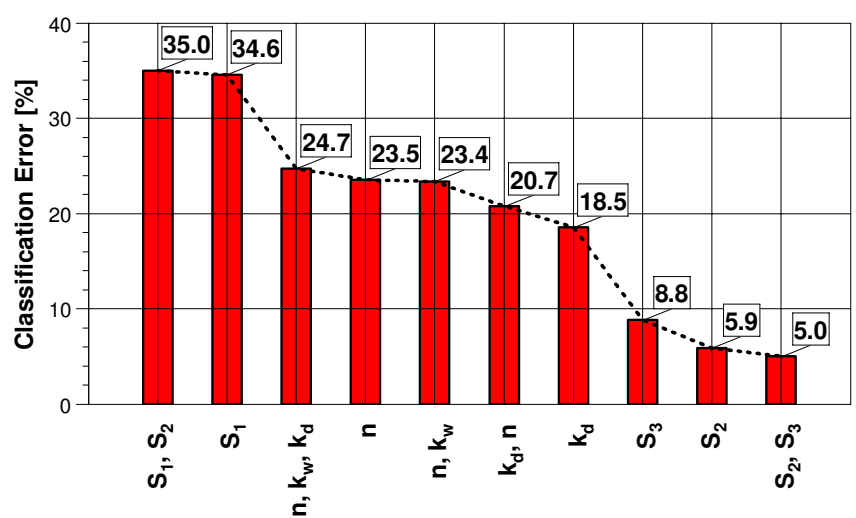

Fig. 9. Feature comparison. Classification error for different feature compositions. A subset of the proposed reflectance features $\left(S_{2}, S_{3}\right)$ clearly outperform any classification based on the extended Oren-Nayar model parameters, exclusively.

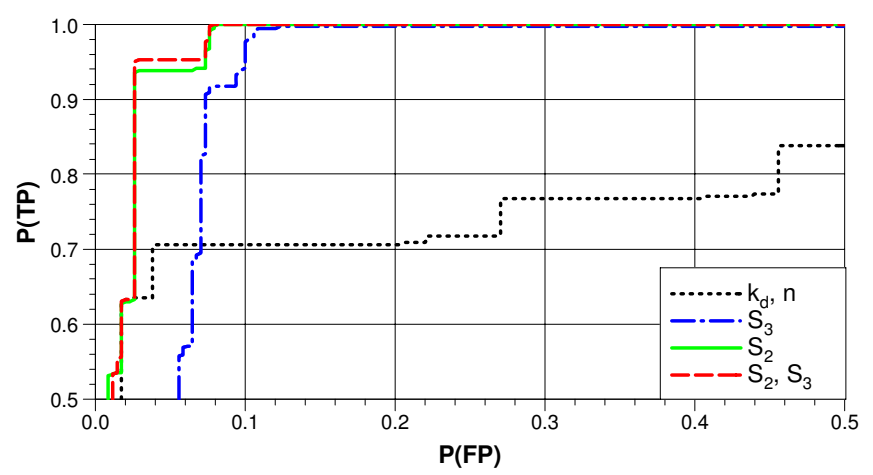

Fig. 10. ROC curves. ROC curves for classification results with $\mathrm{ON}_{\mathrm{ext}}$ parameters and proposed reflectance features.

Since the reflectance parameter $S_{2}$ considers all BRDF values of the estimated $\mathrm{ON}_{\mathrm{ext}}$ model, it is the most stable, single feature for our classification task (error rate: 5.9\%).

Although $S_{1}$ enjoys great popularity in lighting design, it is inapplicable for our application. A classification with $S_{1}$ results in an error rate of $34.6 \%$. Even a combination of $S_{1}$ with $S_{2}$ does not yield any classification improvements. This is due to the reason, that the position of light source and observer evaluated in $S_{1}$ is chosen for the special case of road lighting design and hence is not ideal for road reflectance identification, where the predominant illuminant is the sun.

A classification improvement could be achieved with $S_{3}$ that we individually tailored for our application. Although, this feature is error-prone to measurement noise because it evaluates only a single BRDF value in a defined direction, the strengths of $S_{3}$ appears when combining it with other, stable features. The best classification results are obtained using $S_{3}$ in combination with $S_{2}$ (error rate: $5.0 \%$ ).

We investigate the results in Fig. 9 by computing the ROC curves of selected classification results (Fig. 10). They emphasize the advantage of choosing reflectance parameters rather than classifying road surfaces with $\mathrm{ON}_{\text {ext }}$ model parameters, directly. 

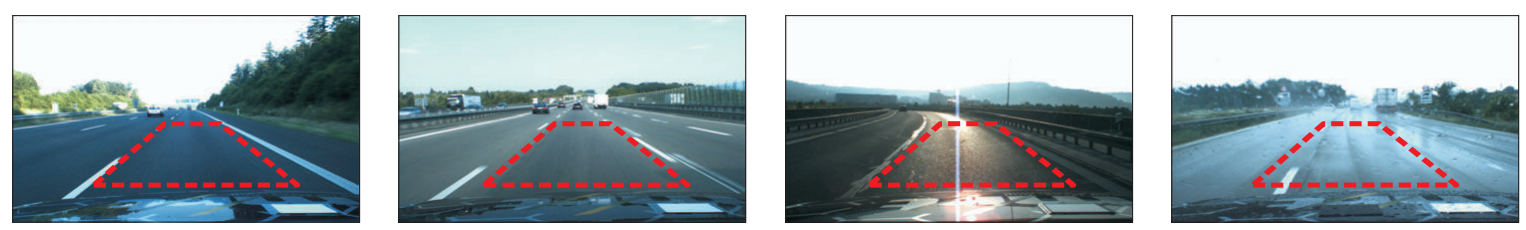

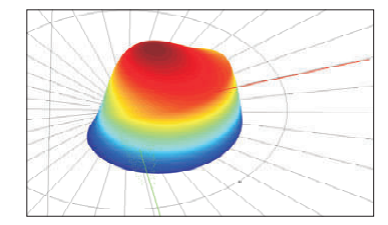

ON $_{\text {ext }}$ Parameters

\begin{tabular}{|c|c|}
\hline \hline$k_{d}$ & 0.42 \\
$k_{w}$ & 0.37 \\
$n$ & 1.00 \\
\hline
\end{tabular}

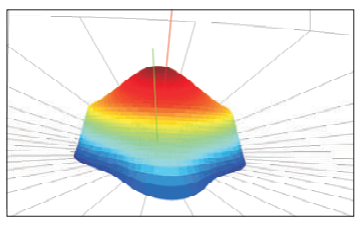

ON $_{\text {ext }}$ Parameters

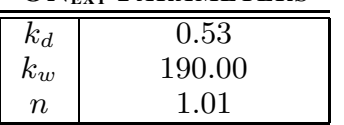

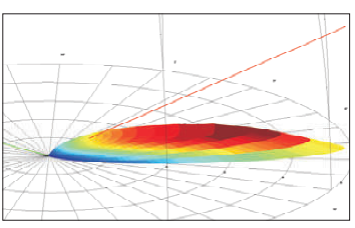

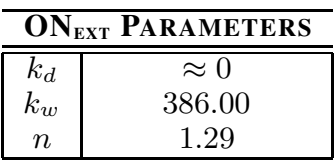

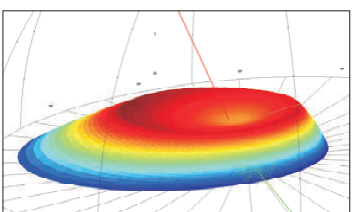

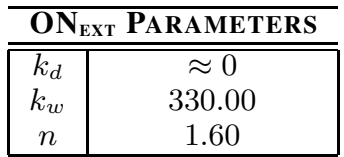

Fig. 7. BRDF model parameter estimation. BRDF measurements are acquired in trapezoidal regions on real road surfaces with diffuse and specular reflectance behavior (top row). The corresponding BRDF models show, that diffuse road surfaces predominantly appear Lambertian whereas for specular road surfaces a preferred direction of light reflections is noticeable (middle row). For well-posed examples, a distinction between the different road surface reflectivity states can be performed, using the extended Oren-Nayar model parameter (bottom row).

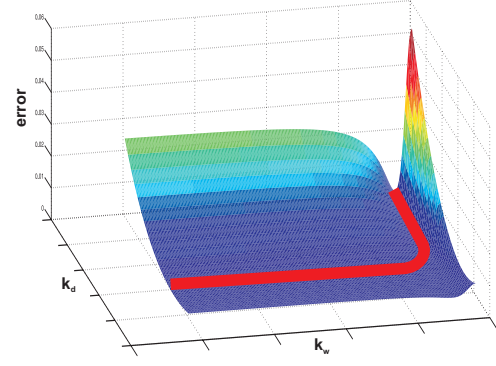

(a) SSD error for different BRDF models.

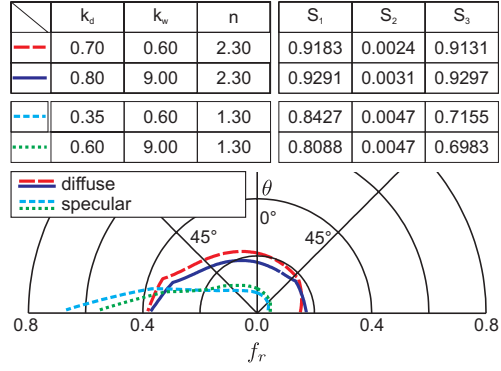

(d) BRDF model ambiguity.

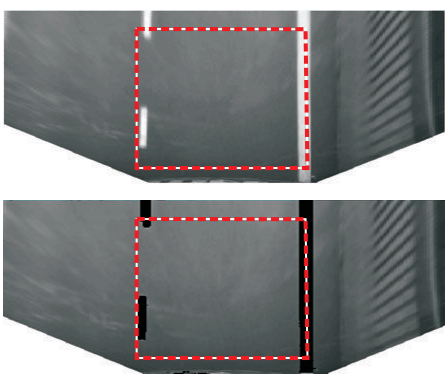

(b) Road markings elimination.

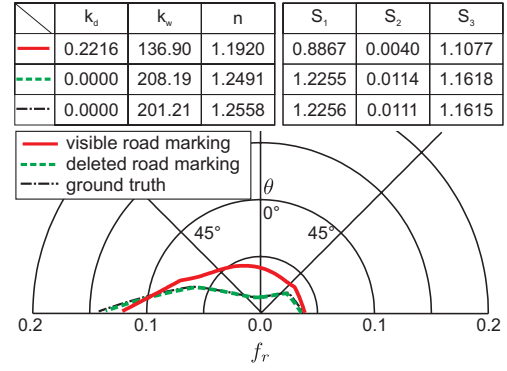

(e) Influence of road markings.

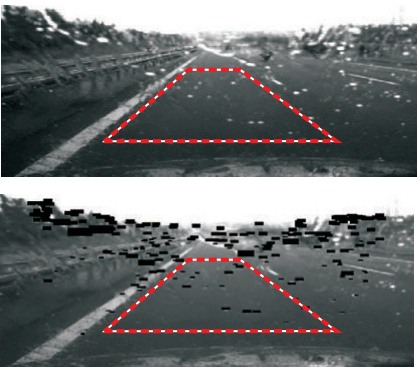

(c) Raindrop elimination.

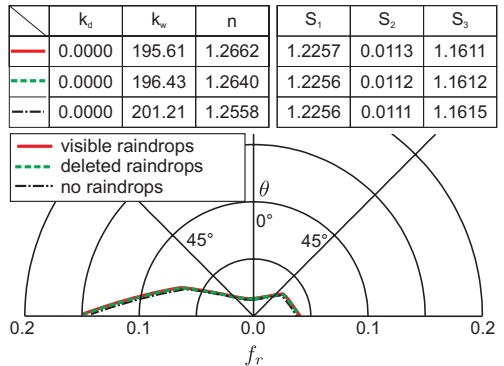

(f) Influence of raindrops.

Fig. 8. Influence of model ambiguities and measurement noise. Model ambiguities arise from the phenomenon, that different $\mathrm{ON}_{\mathrm{ext}}$ model parameters create similar BRDF envelopes with small SSD error (a). For two exemplary parameter sets, their BRDF bodies appear similar, although $k_{d}$ and $k_{w}$ differ significantly (d). However, customized reflectance features $S_{1}, S_{2}, S_{3}$ are more robust and show comparable results (d). Measurement noise, stemming from road markings (e) or adverse weather conditions (f) can influence the BRDF model estimation. These effects can be improved by appropriate preprocessing, like road marking elimination (b) or raindrop detection (c).

TABLE I

CLASSIFICATION RESULTS FOR SELECTED FEATURE. ROWS CONTAIN THE CORRECT CLASSES, COLUMNS THEIR CLASSIFICATION RESULTS.

\begin{tabular}{|c|cc|cc|ccc|cc|}
\hline \multicolumn{2}{c}{$\left(k_{d}, n\right)$} & \multicolumn{2}{c}{$\left(S_{2}\right)$} & \multicolumn{2}{c}{$\left(S_{3}\right)$} & \multicolumn{2}{c}{$\left(S_{2}, S_{3}\right)$} \\
\hline \hline & diffuse & specular & diffuse & specular & diffuse & specular & diffuse & specular \\
\hline diffuse & 205 & 135 & 325 & 15 & 312 & 28 & 331 & 9 \\
specular & 6 & 334 & 25 & 315 & 32 & 308 & 25 & 315 \\
\hline
\end{tabular}

Total error rate: $20.74 \%$ (correct: 539, wrong: 141)
Total error rate: $5.88 \%$ (correct: 640, wrong: 40)
Total error rate: $8.82 \%$ (correct: 620, wrong: 60)
Total error rate: $5.00 \%$ (correct: 646, wrong: 34) 
TABLE II

COMPUTATIONAL TIMES FOR ROAD REFLECTIVITY IDENTIFICATION

\begin{tabular}{|r|l|}
\hline PART & TIME [S] \\
\hline \hline Irradiance estimation & 0.102 \\
Sun position determination & 0.034 \\
Radiance measurement & 0.213 \\
BRDF computation & 0.372 \\
ON ext $_{\text {model parameter estimation }}$ & 1.723 \\
Reflectance parameter extraction & 0.336 \\
SVM classification (test) $)^{1}$ & 0.021 \\
\hline Total CPU time & 2.801 \\
\hline C C++ Matlab Wrapper
\end{tabular}

Despite the satisfactory low-error classification results, difficulties may arise if the assumptions of surface isotropy or incident irradiance constancy are violated. An anisotropic surface behavior may occur because of polished regions on the concrete pavement or minor road surface corrections. Cast shadows may corrupt the assumption of a globally constant incident irradiance. This would lead to irradiance estimates, received from the car hood, that do not represent the actual illumination condition on the road surface anymore. However, both difficulties can be tackled by considering temporal consistency of the measurements.

\section{Computational Times}

We implemented the proposed framework in MATLAB $^{\text {TM }}$ with no special focus on performance optimization. The computational times in Table II are achieved computing the mean value for ten evaluation runs, using an Intel ${ }^{\circledR}$ Pentium ${ }^{\circledR} \mathrm{E} 2180$ with $3.0 \mathrm{GHz}$ and $2 \mathrm{~GB}$ RAM. The mean processing time is 2.8 seconds per image.

\section{CONCLUSion AND Future Work}

We proposed a novel road reflectivity classification approach based on estimating the bidirectional reflectance distribution function of road surfaces. We utilize an extended Oren-Nayar model that considers diffuse and specular reflection characteristics. Meaningful reflectance features allow for an accurate and robust classification of the road reflectance, even in adverse weather conditions. Since we only use GPS information and single gray value images acquired from an uncalibrated camera on board a moving vehicle, the proposed framework is applicable for real-world scenarios and provides valuable information for all driver assistance systems.

Future work will include a detailed evaluation of customized reflectance features. Image sequence analysis will further improve the BRDF model estimation and road surface classification, especially in situations, where the assumptions of surface isotropy or incident irradiance constancy are violated.

\section{ACKNOWLEDGMENT}

This work is partly supported by Honda R\&D Europe. The authors would also like to thank the Karlsruhe School of Optics and Photonics (KSOP).

\section{REFERENCES}

[1] CIE 132-1999. Design methods for lighting of roads. Technical report, Commission Internationale de L'Eclairage, 1999.

[2] J.F. Blinn. Models of light reflection for computer synthesized pictures. SIGGRAPH Comput. Graph., 11(2):192-198, 1977.

[3] R.L. Cook and K.E. Torrance. A reflectance model for computer graphics. ACM Transactions on Graphics, 1(1):7-24, 1981.

[4] K.J. Dana, B. Van-Ginneken, S.K. Nayar, and J.J. Koenderink. Reflectance and Texture of Real World Surfaces. ACM Transactions on Graphics, 18(1):1-34, Jan 1999.

[5] R.O. Duda, P.E. Hart, and D.G. Stork. Pattern classification. Wiley, 2. ed. edition, 2001.

[6] J. Halimeh and M. Roser. Raindrop detection on car windshields using geometric-photometric environment construction and intensitybased correlation. In IEEE Intelligent Vehicle Symposium (IV '09), Xi'an, China, 2009.

[7] E. Hecht. Optics. Addison-Wesley Pub. Co., 4. ed. edition, 2002.

[8] M.J. Jackett and W.J. Frith. Measurement of the reflection properties of road surfaces to improve the safety and sustainability of road lighting. NZ Transport Agency research report, 383, 2009.

[9] B. Jähne. Digital Image Processing. Springer, 6th revised and extended edition edition, 2005.

[10] D.B. Kim, K.S. Part, K.Y. Kim, M.K. Seo, and K.H. Lee. Highdynamic-range camera-based bidirectional reflectance distribution function measurement system for isotropic materials. SPIE Optical Engineering, 48(9), 2009.

[11] W. Leins and P. von Berg. Reflexionsverhalten von Fahrbahnbelägen. Forschungsberichte des Landes Nordrhein-Westfalen ; 2752. Westdeutscher Verlag, Opladen, 1978.

[12] D. Marquardt. An algorithm for least-squares estimation of nonlinear parameters. SIAM Journal of Applied Mathematics, 11:431-441, 1963.

[13] S.E. Marschner, E.P.F. Lafortune, S.H. Westin, K.E. Torrance, and Greenberg D.P. Image-based brdf measurement. Applied Optics, $39(16), 2000$.

[14] W. Matusik, H. Pfister, M. Brand, and L. McMillan. A data-driven reflectance model. ACM Transactions on Graphics, 22(3):759-769, 2003.

[15] G. Meister. Bidirectional Reflectance of Urban Surfaces. PhD thesis, Universität Hamburg, 2000.

[16] S.K. Nayar and M. Oren. Visual appearance of matte surfaces. Science, 267:1153-1156, 1995.

[17] F.E. Nicodemus, J.C. Richmond, J.J. Hsia, I.W. Ginsberg, and T. Limperis. Geometrical considerations and nomenclature for reflectance. Technical report, US Department of Commerce, National Bureau of Standards, Washington, D.C., 1977.

[18] M. Oren and S.K. Nayar. Seeing beyond lambert's law. In IEEE European Conference on Computer Vision (ECCV '94), pages 269280. Springer, 1994.

[19] M. Oren and S.K. Nayar. Generalization of the lambertian model and implications for machine vision. International Journal of Computer Vision, 14:227-251, 1995.

[20] M. Roser and A. Geiger. Video-based raindrop detection for improved image registration. In IEEE Workshop on Video-Oriented Object and Event Classification (in conjunction with ICCV '09), 2009.

[21] C. Schlick. A customizable reflectance model for everyday rendering. In Eurographics Workshop on Rendering, pages 73-83, 1993.

[22] K.E. Torrance and E.M. Sparrow. Theory for off-specular reflection from roughened surfaces. Journal of the Optical Society of America, 57(9):1105-1114, September 1967.

[23] T.C. van Flandern and K.F. Pulkkinen. Low-precision formulae for planetary positions. The Astrophysical Journal Supplement Series, 41:391-411, November 1979.

[24] Bram van Ginneken, Marigo Stavridi, and Jan J. Koenderink. Diffuse and specular reflectance from rough surfaces. Appl. Opt., 37(1):130139, 1998.

[25] A. von Hoffmann. Lichttechnische Anforderungen an adaptive Kraftfahrzeugscheinwerfer für trockene und nasse Fahrbahnoberflächen. $\mathrm{PhD}$ thesis, TU Ilmenau, 2003.

[26] H. Wambsganß. Bestimmung und messtechnische Erfassung des Reflexionsverhaltens von Fahrbahnoberflächen bei kfz-eigener Beleuchtung. Forschung Straßenbau und Straßenverkehrstechnik ; 699. Bundesminister für Verkehr, Abteilung Straßenbau, Bonn-Bad Godesberg, 1995.

[27] G.J. Ward. Measuring and modeling anisotropic reflection. SIGGRAPH Computer Graphics, 26(2):265-272, 1992. 